\title{
Théologiques
}

\section{Le reliquat des chagrins : essai sur le rituel du deuil}

\section{Christian Saint-Germain}

Volume 2, numéro 2, octobre 1994

L’esprit

URI : https://id.erudit.org/iderudit/602410ar

DOI : https://doi.org/10.7202/602410ar

Aller au sommaire du numéro

Éditeur(s)

Faculté de théologie de l'Université de Montréal

ISSN

1188-7109 (imprimé)

1492-1413 (numérique)

Découvrir la revue

Citer cet article

Saint-Germain, C. (1994). Le reliquat des chagrins : essai sur le rituel du deuil. Théologiques, 2(2), 117-127. https://doi.org/10.7202/602410ar d'utilisation que vous pouvez consulter en ligne.

https://apropos.erudit.org/fr/usagers/politique-dutilisation/ 
Théologiques 2/2 (1994) 117-127.

\title{
Le reliquat des chagrins : essai sur le rituel du deuil
}

\author{
Christian SAINT-GeRMAIN \\ Département des sciences religieuses \\ Université du Québec à Montréal
}

"L'année s'en va j'ai caché à mon père mes cheveux gris. " (Etsujin) ${ }^{1}$

Cette communication se propose de tenter de comprendre l'efficace des rituels pour initier au processus du deuil. Elle questionne d'abord la distinction maintenant classique entre deuil et mélancolie, établie principalement en 1917 par un article important de Freud, intitulé justement "Deuil et mélancolie". Ensuite, il s'agira d'apprécier l'espace rituel laissé vacant par le mourir dans la communauté moderne. Que signifie ce blanc, ce noir, ce vide où personne ne sait plus son rôle? Peut-on faire l'économie de la duplicité d'une mise en scène sociale pour conjurer la violence d'événements aussi importants dans la vie des individus? Les sociétés traditionnelles ont, dans le passé, par le biais de la religion, fourni mots et gestes pour vivre socialement la perte, la faire accepter aux proches, la porter socialement. Dans un contexte d'effacement de l'inscription institutionnelle du religieux, peut-on vivre la mort autrement que dans le silence, le malaise? Perdre le rituel, c'est perdre le sens du deuil qui se situe justement à la limite du lien, du discible, de l'intolérable. Une manière de faire-avec l'intolérable ou la jouissance d'une perte d'objet. Or, le lieu rituel quel qu'il soit donne une scène à cet affect douloureux, le convoque, l'appelle, et c'est l'histoire de bien des endeuillés que de ne trouver consolation, de n'avoir pu accuser significativement une perte. Cet exposé vise a mettre en relief le sens de la transaction rituelle et à comprendre les effets, dans l'économie psychique des individus, de son absence. Une manière de se demander si l'on peut penser la douleur du perdre sans le secours des représentations religieuses. À cet égard, on ne 
peut certes guérir de la mort, mais l'on doit continuer d'entendre la vie à travers elle.

\section{Attachement et séparation}

De ses parents, l'enfant reçoit la vie mais aussi pour les voir disparaître, eux, pure source de silence d'avant sa vie, cercle plus grand encore que l'enveloppe des voix aimantes, des mots qui l'ont vêtu, des mains venues le border au soir des premières terreurs. La mort des parents confine à la reconnaissance d'une mémoire archaïque, force à un égoïsme très primitif, rappelle à la doublure intime d'une solitude fœtale. Rupture qui rappelle peut-être pour l'enfant au lointain celle de se détacher de la parois utérine, d'entreprendre ce voyage. Ce jeu avec l'absent se déroule ainsi pour l'enfant à la lisière, sur la fibre de toutes les possibilités d'attachements futurs, il répète la scène d'une vie à deux devenue brusquement aller simple vers sa fin. Difficile apprentissage que cette solitude primordiale avec laquelle le sujet aura pourtant à composer toute sa vie. C'est le travail du petit tout occupé à se doter d'objets transitionnels, se mobilisant pour affronter les départs, les séparations, les espaces où ils se retrouvent sans attention adulte. Cette capacité de s'affronter à l'absence de stimulation requiert de la part du futur adulte un capital de soins, de regards, d'affections, de traitements agréables qui lui ont laissé le goût d'investir le réel mais aussi la possibilité de s'en proréger. Le psychanalyste Donald Winnicott a résumé, cette situation, en 1958 dans un article intitulé "la capacité d'être seul " 2 . Dans cet article, Winnicott établit un lien entre cette capacité d'être seul et les rapports étroits qu'entretient un individu avec les souvenirs agréables de ses premiers objets d'amours, point d'ancrage de sa personnalité. Ainsi notait-il :

La relation de l'individu avec ses objets internes, qui va de pair avec une confiance dans ses relations internes, fournit à elle seule une raison suffisante de vivre, si bien que, temporairement, il est capable d'être heureux, même en l'absence d'objets et de stimulations externes. La maturité et la capacité d'être seul impliquent que l'individu a eu la chance, grâce à des soins maternels suffisamment bons, d'édifier sa confiance en un environnement favorable. Il y est

2 Donald WINNiCOTT, De la pédiatrie à la psychanalyse, Paris, Payot, 1969, p. 205-213. Mélanie KLEIN a aussi réfléchi dans cette direction à l'occasion d'un article de 1963 intitulé « Se sentir seul » dans Envie et gratitude et autres essais, Paris, Gallimard, 1968, p. 120-137. 
parvenu par la répétition de gratifications instinctuelles satisfaisantes ${ }^{3}$.

\section{Où le sujet négocie avec ce qu'il ne peut perdre}

Une illusion motrice mobilise donc ainsi l'appareil psychique qui souhaite voir réapparaître, de retard terrifiant en délai consenti, par la répétition, le retour de l'objet satisfaisant. Cet itinéraire est celui-là même du symbole, de la réparation, de la construction artistique. D'ailleurs, pour reprendre une formule théologique qu'Augustin utilisait à propos de la recherche de Dieu, si l'objet n'avait pas en quelque façon déjà été trouvé, le sujet ne le chercherait pas autant. Dans ce contexte, l'amour n'est pas une découverte mais une forme singulière de reconnaissance, de retrouvailles. Mais ce début timide, par lequel le moi immature prend appui sur celui de la mère, pour, dans des conditions normales, s'en émanciper, n'explique pas à lui seul cette naissance à rebours qu'est le processus de deuil. Du point de vue analytique, l'autonomie s'acquiert dans la scansion fluide de la présence et de l'absence, dans l'évolution normale des attachements successifs. Toujours dans cette optique, le moi se voit engagé à prendre des décisions vitales, à retirer le gros de son transport avant d'être lui-même emporté par l'objet perdu (dans le monde des affaires : éviter un "reverse take over"). D'où le sens du deuil qui consiste à tuer la chose, l'objet dans lequel s'étaient engluées, s'étaient étayées des couches d'investissements. Une manière de revenir contre l'objet avant d'en être hanté, possédé, d'en expulser le cadavie pour en éviter le fantôme. Mais on le comprendra, l'opération n'est pas si simple, cet abandon ne se laisse pas ramener à un déguerpissement, à sauver les meubles sous les cendres du moi. Pour Freud, dans un texte connu et profond intitulé "Deuil et mélancolie ", le sauvetage du moi prend la forme d'un repli stratégique, une manière lente et énigmatique de se déprendre, de réorganiser l'économie du moi en récession. Freud écrit :

De même que le deuil amène le moi à renoncer à l'objet en déclarant l'objet mort, et de même qu'il offre au moi la prime de rester en vie 4 , de même chacun des combats ambivalentiels singuliers relâche la fixation de la libido à l'objet en le dévalorisant, en le rabaissant et même, pour ainsi dire, en le frappant à mort ${ }^{5}$.

D. WINNICOTT, De la pédiatrie à la psychanalyse, p. 208.

Le souligné est de nous.

5 Sigmund FrEUD, Métapsychologie, Paris, Gallimard, 1940, p. 172 


\section{La douleur théorique}

Ce modèle à l'avantage de ramener à un tableau clair des opérations complexes du moi pour se délester de l'objet aimé. Toutefois, ce retournement qui s'appuie sur une manière de revisiter l'ambivalence initiale des sentiments à l'égard de l'objet n'arrive pas à faire voir où se prend cette salutaire décision, où commence pour le moi cette reprise de possession forcée de lui-même, et comment il retrouve, dans le meilleur des cas, pratiquement intact ses actifs. En fait, il est ardu d'imaginer dans le champ des représentations de l'appareil psychique comment le mécanisme se démonte. C'est comme si dans ce modèle, Freud suggérait qu'il suffisait de reprendre, une à une, ses billes, d'emprunter en sens inverse la transaction amoureuse. Comme si le deuil retrouvait dans la haine de l'objet son salut et que la mélancolie menaçait le moi précisément à chaque fois où le deuil échouait à supprimer les adhérences de et à l'objet. Dans ce contexte, le deuil met à jour l'ambiguiité des attachements, leur caractère superficiel par rapport au désir narcissique de faire du même. Cela laisse pourtant sans explication les motifs préliminaires, les prédispositions inconscientes qu'avait eu le moi d'épouser la forme particulière de l'objet aimé, les motifs pour lesquels il s'était, dans un premier temps, identifié à lui. Mais le médecin viennois s'empresse toutefois d'ajouter :

Il est vite dit, et il est facile d'écrire, que la représentation (de chose) inconsciente de l'objet est abandonnée par la libido. Mais en réalité cette représentation figure sous la forme d'innombrables impressions particulières (traces inconscientes de celles-ci) et l'accomplissement de ce retrait de la libido ne peut être un processus instantané; c'est certainement, comme le deuil, un processus de longue durée progressant pas à pas. Il n'est certainement pas facile de distinguer s'il commence simultanément en plusieurs endroits ou s'il comporte une série qui serait déterminée $(\ldots)^{6}$.

Il s'agit, à n'en pas douter, d'un travail, d'une souffrance pour extraire la somme des excitations, neutraliser les souvenirs plaisants, qu'a déposé dans le moi, l'objet élu. Et la colère produite par l'absence de l'objet n'est pas suffisante pour réduire la fracture que laisse ouverte sa déconvenue. Et c'est la litanie des chagrins prononcée en silence par le moi en mal de l'Autre, encore accroché à lui. Sobre douleur des premiers moments où l'objet manque à l'appel, rage impuissante que de ne le point trouver, tout 
en s'y trouvant captif. Douleur aussi vieille que la petite peine d'un enfant morose. Comme le note fort justement Julia Kristeva :

Cette tristesse est le filtre ultime de l'agressivité, la retenue narcissique de la haine qui ne s'avoue pas, non par simple pudeur morale ou surmoïque, mais parce que dans la tristesse le moi est encore confondu avec l'autre, il le porte en soi, il introjecte sa propre projection omnipotente et en jouit. Le chagrin serait ainsi le négatif de l'omnipotence, l'indice premier et primaire que l'autre m'échappe, mais que le moi, cependant, ne s'accepte pas abandonné?

\section{Le reliquat des chagrins}

C'est tout l'imaginaire de l'addiction, de la toxicomanie qu'implique le travail du deuil, ce sevrage où le moi se dégrise péniblement de sa confiance dans le monde extérieur, sans pour autant y renoncer définitivement. Il a incorporé l'objet aimé et l'en voilà tout retourné. Est-ce la plainte de la mère dépossédée qui hante le sujet au fond de ses douleurs, qui souffre en lui? Quoi qu'il en soit, ce qui demeure proprement mystérieux est la manière dont le moi va conserver à travers les méandres de ses mésaventures, l'impulsion à remplacer par d'autres objets l'espace d'investissement laissé vacant en lui. Et l'on prendra peut-être trop rapidement pour de la maturité, le fait que le sujet, d'une fois à l'autre, voit diminuer sa capacité d'aimer, rétrécir cette possibilité d'abandon, voit se substituer à l'amour véritable, un enchaînement à des solutions de compromis. Pour conserver la métaphore économique, les investisseurs qui ont été témoins du krach n'ont plus jamais, dit-on, été les mêmes. Ils ont perçu le revers de l'investissement, qui n'est même plus le contre-investissement ou le retour sur l'économie, mais le point d'horreur anomique. Dans la perspective où s'accomplit un deuil simple, la mort d'un proche, tout peut reprendre avec la prime pour le moi de "rester en vie ". Que dire alors de la perte de l'objet aimé, resté vivant. Comment en faire le deuil, en revenant au sentiment ambivalent qui présidait à son choix, peut-être, mais seulement peut-être. Tout se passe comme si l'Autre n'était que le prétexte à un envoi narcissique qui pouvait se retrouver, à défaut de retour, en poste restante. Mais c'est que pour l'aimer, ou l'élire, le moi a dû s'aliéner dans son objet, se perdre en lui, y placer tout son capital d'idéalisation et, éventuellement, le sentiment inimaginable sur lequel se fonde sa propre existence. Il a dû voir dans l'Autre une possibilité d'expansion impérialiste, un agrandissement du moi, mais aussi l'investir de toute la consistance 
imaginaire qui le protège lui-même contre la dureté du réel ${ }^{8}$. C'est la force de ce transfert que la résorption dans le modèle économique n'arrive pas à décrire. Freud en est sans doute conscient lui qui rapprochait justement le maniement du transfert avec celui des explosifs. À cet égard, il ne suffit pas d'éteindre, tel un écran d'ordinateur, l'appareil psychique pour que s'évanouisse comme par miracle l'ombre de l'objet. Ni de saisir avec des mots, ou à l'occasion d'une théorie, l'alchimie étrange, la magie lente par lequel le moi se libère de son identification envoûtante. Autant dire qu'il suffirait de se déclarer en amour (ou se déclarer solennellement souverain sic...) pour en éprouver le sentiment océanique. La théorisation est un rituel nécessaire mais bien approximatif. L'expérience freudienne établit pourtant une ligne de partage nette entre le deuil et la mélancolie précisément à chaque fois où la perte ne peut s'accuser; où l'Autre persécute son hôte. Adoptant la perspective freudienne, Marie-Claude Lambotte écrit :

Le processus dynamique de la mélancolie atteste (...) cette impossibilité pour le sujet de se séparer de l'objet perdu et de réinvestir son énergie ainsi libérée sur un substitut. Mais alors que le deuil s'achève après un temps plus ou moins long, la mélancolie s'installe au contraire, sous la forme de l'incorporation de l'objet perdu au sein du sujet lui-même de telle manière que celui-ci reprend à son compte l'ambivalence des sentiments qu'il portait auparavant à l'objet aimé 9 .

Et c'est une histoire bien connue que cet effondrement du moi, de son appauvrissement sous le régime d'un Autre enkysté en lui. L'identification à l'Autre aimée a achoppé, cette blessure d'amour insituable va drainer, telle une incision secrète, la libido du moi. Le moi est souffrant d'un point qu'il ne peut appréhender - et conséquemment dont il ne peut guérir et en subit l'impact balistique.

Le processus peut être ainsi reconstitué : d'abord un choix objectal, c'est-à-dire un rattachement de la libido à une certaine personne, puis, un relâchement de la relation objectale sous l'influence d'une offense ou d'une déception de l'Aimé. La libido ne se déplace pas sur un

8 Comme le note Jean OURY, «On ne peut vivre que protégé par l'imaginaire et cette sorte d'enveloppe est nécessaire pour la mise en forme, la mise en consistance de toute existence », "Violence et mélancolie » dans La violence. Actes du colloque de Milan. 1977, (A. Verdiglione, dir.), Paris, Union générale d'édirions, 1978, p. 25.

9 Marie-Claude LAMBOTTE, Esthétique et mélancolie, Paris, Aubier, 1984, p. 32. 
autre objet, elle se retire dans le moi, où elle sert à établir une identification du moi avec l'objet abandonné ${ }^{10}$.

\section{Le travail de perdre}

Le moi s'adresse à lui-même des reproches qui sont la tentative maladroite d'atteindre l'Autre en soi, de le tuer. La plénitude du sentiment amoureux doit faire place à la difficile gestation d'une colère efficace. Au passage d'un plein à l'endeuillement, à l'acceptation d'être abandonné, déshabité, d'entrer en travail. Cette désorganisation n'est pas étrangère au processus créateur, à la crise déstructurante qui consiste dans un même moment à se soustraire à un Surmoi stérilisant, et à s'en faire aimer par l'adresse nouvelle qu'implique la création ${ }^{11}$. Elle n'est pas étrangère non plus au moment même où le sujet s'est perdu dans l'Autre, et se cherche maintenant dans le deuil. Quelque chose de plus que lui-même, qui transitait par lui - inspiration, moment de grâce - sans lui appartenir en propre, qu'il vit désormais comme une perte déterminante. Violence subtile, effraction, mais surtout retournement car, comme le note Daniel Sibony: "Tout ce qui touche à l'amour, donc à l'inconscient, est voué au paradoxe, c'est-à-dire au retournement qui le ramène à son contraire. ${ }^{12}$ " Cette ambivalence est bien le chiffre d'un ressenti au cœur du moi, de la préséance dans le développement des individus de la morsure sur le baiser. C'est à théoriser cette valse hésitation qu'apparaissent des notions comme le deuil et la mélancolie, à penser les restes d'une violence impossible à liquider que l'appareil théorique se met en branle. La théorie n'est-elle pas d'ailleurs une forme particulière de la ritualité occidentale? Une manière de ressaisir les passages à vide, les tremblements, de stabiliser dans une chorégraphie rationnelle les instants de panique? De ne rien perdre de la perte et de promettre une lumière sans ombre? Il s'agit d'une façon d'observer à l'intérieur d'un champ conceptuel une transaction présumée dans le réel, de l'en extraire suffisamment, pour qu'elle

Jacques LACAN (dir.), Scilicet, Les articles de la revue Scilicet ne sont pas signés, « Signification de la mort par suicide », Paris, Seuil, 1968, p. 142.

11 Comme l'indique Didier ANZIEU dans son important ouvrage Le corps à l'œuvre: «Un des paradoxes du travail créateur réside dans l'espoir de se faire aimer un jour sinon de son Surmoi, du moins d'un des personnages intériorisés qui composent celui-ci. C'est un paradoxe, car sans un amour suffisant du Surmoi on ne saurait devenir créateur, (...) et sans l'accomplissement d'une œuvre dont la valeur soir reconnue, on ne saurait forcer l'amour du Surmoi ». Paris, Gallimard, 1981, p. 115-116.

12 Daniel SıBONY, L'amour inconscient. Au-delà du principe de séduction, Paris, Grasset, 1983, p. 182. 
apparaisse satisfaisante au regard de la raison. Mais cette manière toute extérieure d'expliquer un phénomène, de décrire un processus, dans le cas de la rhétorique psychanalytique à l'égard du deuil et de la mélancolie, est déjà une voie suggestive de guérison pour le patient. $Y$ aurait-il un chamanisme conceptuel dans la croyance partagée de ses utilisateurs? Mais cela n'aurait rien de diminutif puisqu'il s'agit de penser ici rien de moins que le poids désorganisant, la violence non symbolisable des départs inouiis. En fait, de repasser par la trame des liens parentaux, de comprendre le sens du vivant. Dans ce contexte, le rituel religieux s'est présenté d'abord comme un expédient symbolique, un contrat passé avec l'immense, une manière de faire-avec la vie et la mort.

\section{La fine doublure des douleurs : la ritualité}

Toutefois, le faire du rituel n'est pas résumé par la production, il est tout entier convocation de l'extrême dans le peu. Et dans le cas des rituels de deuil, une façon de se faire reconnaître par les autres en conservant sur soi des signes visibles, vestimentaires, pendant un laps de temps déterminé. L'équivalent peut-être du temps de parturition auquel succède, pour les femmes dans les sociétés traditionnelles, une période de retrait. Non pas l'usage contemporain d'une parole bavarde qui s'enquiert avidement des états d'âme du survivant, mais une distance respectée. Il s'agit de décrire une retenue qui n'est pas l'inhibition, une politesse qui ne cache pas une indifférence codifiée. Était-ce le sens que convoquait ce bref poème japonais, cet haïku laissé ici en exergue : "L'année s'en va j'ai caché à mon père mes cheveux gris "(Etsujin)? Suprême politesse que de ne laisser voir à cet Autre contre lequel ma vie s'est déjà reposée, que la sienne est presque entièrement passée dans la mienne. Étrange capture d'une émotion fixée sur la pellicule des mots, suave plongée vers un malaise ressenti par l'enfant à la vue d'un temps pressenti. Les parents s'éloignent imperceptiblement dans l'exacte mesure où leurs enfants s'en approchent, inquiets de se sentir doublement mourir, de s'affronter euxmêmes à ce sourire négatif. Aucun lien ne dure sinon pour ne s'exercer en fait qu'à se refermer sur le quotidien de toute les solitudes. Il est difficile pour les enfants de ne pas être emportés par l'impuissance de ceux en qui, pourtant, tous les rêves de puissance, de protection contre la dureté du monde, avaient été jadis placés. À cet égard, les institutions, les idéologies, viennent s'insérer au lieu qu'occupait pour l'enfant, l'image de ses parents. Comme le note à ce propos, le psychanalyste Didier Dumas :

Le travail du deuil est douloureux à tout âge. Il n'y a pas de désespoir plus grand, pour l'enfant, et plus tard pour l'instance infantile restée en adulte, que de constater son impuissance à aider ses parents 
au lieu de leur impasse. Il faut bien pourtant supporter que la fonction maternelle, ne soit, aussi, qu'un lieu de passage, une symbolique de l'entrée et de la sortie ${ }^{13}$.

Dans ce contexte, il n'est pas étonnant que cette symbolique de l'entrée et de la sortie soit celle-là même que reprend l'Église lors de la cérémonie du baptême et lors des funérailles, dans l'aspersion symbolique des eaux, de la rupture des eaux à la naissance jusqu'au retour dans le giron de la terre. Lecture des textes de toute traversée, de tout bord, de la sortie d'Égypte à l'entrée en terre promise. Du début de la vie jusqu'à sa fin, qu'un peu d'eau reçue dans toute une remise en scène de sortie de la mère et de retour en elle. Maternage, toilette qui s'adressent tout aussi bien à l'enfant naissant qu'au corps des défunts. Une manière de dire le trajet de l'individuation de tout être humain. La mère est appelée au moment de mourir comme à celui de naître. De même, pour l'enfant, faudra-t-il guérir du sentiment d'avoir infligé à sa mère, par sa naissance, les plus vives douleurs, la plus extrême séparation d'avec elle-même. Naissance et deuil s'inscrivent comme dans le souffle d'un même cri de femme, d'enfant. Or, la fonction rituelle ne s'applique pas à se vouloir originale mais bien plutôt rassurante, paroles amniotiques qui bercent les douleurs, rétablissent un contact avec le connu à l'occasion du plus périlleux déséquilibre.

Le rituel ne s'exerce pas non plus à penser la mort en soi ni à essayer d'expliquer la vie. Il agit toujours pour moduler collectivement les effets de la mort d'un autre sur soi. Quelqu'un meurt dépositaire du désir, des attentes des vivants, et voilà que le lien tressé au fil des jours, des mots, revient vers ses survivants. Il s'agit d'une situation qui confine chacun à n'éprouver que des vertiges, des émotions devant la force d'un tel appel d'air autour de l'absent. C'est cette brèche inquiétante que viennent colmater des paroles aussi simples que celles qui invitent l'âme des fidèles défunts à reposer en paix.

\section{Les sanglots longs}

Dans le catholicisme, la mort est très tôt comprise comme porteuse d'un instant de vérité, dernière chance pour le moribond de faire amende honorable, d'expier ses fautes ${ }^{14}$, et de laisser aux survivants, le souvenir

13 Didier Dumas, Hantise et clinique de l'Autre, Paris, Aubier, 1989, p. 74.

14 Voir à ce propos, Jean-Thierry MAERTENS, Ritologiques 5 Le jeu du mort, Paris, Aubier Montaigne, 1979, p. 148. 
édifiant d'une fin de vie ordonnée. La mort est récupérée à des fins pastorales, pédagogiques. Il s'agit d'un baptême à l'envers, d'un exorcisme de dernière minute. Toutefois la sagesse du mourir traditionnel cache à peine l'importante explosion d'humeurs qu'annonce l'événement même. $\mathrm{Ni}$ le sentiment que rien n'est définitivement fixé dans le vécu psychique des sujets, que les récentes amours comme les rages de naguère, sont encore portées par les survivants. Est-ce pour cela que résonnent dans les sociétés dites archaïques, les tambours d'afflictions? En fait, dans les rituels, c'est le circuit pulsionnel qui est déconnecté ou repris par une liturgie tellement lente que plus rien d'essentiel ne s'y passe, est-ce étonnant alors que le sens désormais nous échappe? Comme le souligne à ce propos Élias Canetti;

Les gestes du prêtre en ornements lourds et rigides, sa démarche compassée, les paroles qu'il prononce en traînant, tout cela rappelle un peu une lamentation funèbre infiniment subtilisée, délayée sur des siècles avec une telle régularité qu'il n'y reste quasiment plus rien de la soudaineté de la mort, de la véhémence de la douleur : le déroulement temporel de la lamentation funèbre est momifié ${ }^{15}$.

C'est que l'événement de la mort réfute l'enchaînement des significations, fait sortir le sens de ses gonds. À sa violence, les sociétés traditionnelles ont répondu par des rixes, des crises de nerfs et des danses. Ainsi rapporte Jean-Thierry Maertens :

Les paysans Irlandais au siècle dernier achevaient les funérailles dans une mêlée générale où chacun frappait son voisin au signal donné par le plus proche parent du défunt. Chez les Kukuya (Congo), le décès d'un individu est suivi d'une longue palabre sur les causes de sa mort qui débouche à plusieurs reprises sur de réelles bagarres ou en tout cas des mimes chargés d'agressivitét ${ }^{16}$.

On peut soupçonner qu'autant d'exubérance soulageait les individus d'affects (et cela même avant l'arrivé chez le notaire sic...) que bien des heures de thérapie, de paroles filandreuses, n'arriveraient pas encore à libérer. C'est que la meilleure recollection passe par la dislocation, futelle symbolique. C'est sans doute pour cela aussi qu'il a fallu d'abord le Concile de Rouen de 1231 pour interdire, sous peine d'excommunication, la danse dans les cimetières et, jusqu'au $17 \mathrm{e}$ siècle, rappelle J. T. 
Maertens, pour que l'on cesse définitivement la pratique de ces danses macabres.

La danse est dès lors remplacée par le "cortège " d'enterrement dont la solennisation chrétienne apparaît au $12^{\mathrm{e}}$ siècle. Tout y est ordonné, chaque sexe, chaque rôle à sa place, défini à partir du corbillard, la mort assurant encore dans cette ostentation le statut de la famille et ses rapports sociaux 17 .

Mourir implique pour le survivant, un rappel de sa propre venu au monde, elle devrait lui donner l'occasion d'investir ce passage, d'en crier l'étrangeté. Deuil de la séparation, théâtre de la rupture, pourquoi les rites n'en retrouvent plus le lyrisme, la grande santé?

C'est à cette transaction entre morts et vivants à laquelle le rituel devrait répondre. Apaiser les uns et donner aux autres la permission de continuer à vivre. Étrange intersection, fascinante solution de compromis, la mort ne peut s'interdire la mise en scène, un avoir-lieu. Comme l'indique Georges Bataille: "La connaissance de la mort ne peut se passer d'un subterfuge : le spectacle ${ }^{18}$." Essence de tout ce qui se dérobe, mère de toutes les formes de surprise, son approche n'est nullement circonscrite par la connaissance objective. Dans les sociétés traditionnelles, la poésie des rituels a justement pour fonction d'amadouer, de prendre en compte, de délivrer par gestes et mots tout ce potentiel d'absence qui pèse dorénavant sur les sujets. Elle ouvrait aussi un nouvel horizon. Mais c'est aussi un paradoxe pour l'observateur du phénomène religieux de voir dans les sociétés modernes les individus réemprunter, à l'occasion du deuil, le chemin des rites dont le sens s'en est pourtant allé avec leur pratique. On se fait difficilement à l'idée de laisser son semblable mourir sans cérémonie. Il faut récupérer pour soi, derrière les chers disparus, un peu de sens à la vie, pour la vie, fut-il étranger à des définitions précises de la foi.

17 J.-T. MAERTENS, Ritologiques 5 Le jeu du mort, p. 163-164.

18 Mikkel BORCH-JACOBSEN, Lacan le maittre absolu, Paris, Flammarion, 1990, p. 117. 